\title{
Performance Analysis of a Modular Multilevel Converter Drive System with Model Predictive Control
}

\author{
Peng Dai, Zheng Gong ${ }^{*}$ and Guosheng Guo \\ School of Information and Electrical Engineering, \\ China University of Mining and Technology, Xuzhou, Jiangsu 221116, China \\ gzcumt@163.com
}

\begin{abstract}
The modular multilevel converter (MMC) has been widely researched in the past decade for its superior performance in high voltage high power applications. However, applying MMC into electric motor drive systems is a intrinsically challenging problem because the capacitor voltages fluctuate in a wide range when the converter works at low output frequencies. A novel model predictive vector control (MPVC) scheme is presented to ensure the output phase currents' quality of MMC for entire frequency range. In this scheme, model predictive control (MPC) is adopted to track the phase currents' reference values and balance the capacitor voltages as far as possible. By replacing the conventional inner current control loops and pulse width modulation (PWM) module with MPC controllers, the vector control oriented by rotor flux is constructed to meet the requirements of drive systems. Simulations of a MMC drive with an induction motor are carried out using the Matlab/Simulink. The simulation waveforms demonstrate that the drive system achieves satisfied steady and dynamic performance.
\end{abstract}

Keywords: Modular multilevel converter, Model predictive control, Vector control, Drive system

\section{Introduction}

The multilevel voltage source converters (VSC) have been widely used in medium or high voltage high power applications, such as motor drives, power transmission, power quality improvement, and so on [1-3]. Among the various multilevel VSCs, modular multilevel converter (MMC) becomes more and more popular in the past few years, especially in the field of high voltage direct current (HVDC) transmission system [3-7]. When compared to the neutral-point-clamped (NPC) multilevel converters and the flyingcapacitor (FC) multilevel converters [8-10], MMC is easier to achieve high voltage level numbers and being much more modularized. MMC is similar to the cascaded $\mathrm{H}$-bridge multilevel converter (CHMC) to some degree because both of them have the modularized circuit layout $[8,11]$. Taking advantage of the existing of the common dc-link which the CHMC lacks of, MMC is more suitable for the system that needs to operate in a back-toback way. SIEMENS has constructed the world's first HVDC power transmission system based on MMC with the trade name of HVDC PLUS in the year of 2010.

MMC is based on the cascaded connection by a series of submodules (SMs) in each arm. Thus, there are a lot of floating capacitors in the converter. Similar to other VSCs, keeping these capacitor voltages balanced is one of the intrinsically task to ensure the MMC's stable operation. Some research papers proposed that by sorting the capacitor voltages in each arm [12-14], the working states of submodules can be determined according to the arm current's directions. This strategy can be used in different modulation methods, such as multicarrier PWM techniques in [7] and [15] and the submodules unified pulse width modulated (SUPWM) method in [12-14]. Though this 
voltage balancing control strategy achieves a satisfied effect, the dynamic sorting may result in extra switching loss. What's more, when MMC is expended to high number of voltage levels, the sorting algorithm for the numerous capacitor voltages will exacerbate the burden of the digital controllers. Another capacitor voltage balancing control strategy was introduced in [7] for the carrier-phase-shifted PWM (CPSPWM) method. It utilizes three closed-loops to regulate the reference voltage for each arm. The control strategy's parameters become more difficult to adjust as lots of PI controllers were brought in. Reference [14] analyzes the principle of the capacitor voltage fluctuations with theoretical equations. The fluctuations are proportional to the amplitudes of output currents and inversely proportional to the output frequency. So it's unsuitable to apply MMC into drive systems directly. Reference [14] and [16] proposed the method of injecting high frequency common mode voltage components and circulating current components into the arms to suppress the fluctuations at low frequencies directly. The startup performance of induction motors is improved significantly by using this approach based on the conventional V/f control strategy. However, the proposed method is suitable for drives with quadratic torque loads and the implementation in high performance electrical drive control strategies, such as vector control (VC) and direct torque control (DTC) were not adopted.

Model predictive control (MPC) has been developed for power converters and electrical drives in recent years [17-20]. To control the output phase currents of the converter and balance the capacitor voltages synchronously, an MPC method of MMC is developed in this paper. Vector control oriented by rotor flux is adopted to ensure the steady and dynamic characteristics. The details are described by the following parts. In Section 2, the system structure and operating principles of the MMC used in motor drives are introduced. Section 3 analyzes mathematical model of a dc-ac MMC and derives discrete mathematical models of variables for the MPC strategy. Section 4 presents the design of control system. Simulations studies analyses are carried out in the Matlab/Simulink software to investigate the effectiveness of the drive system in Section 5. Conclusions are summarized in Section 6.

\section{System Structure and Operating Principles}

The main circuit configuration of an inverter based on three-phase MMC is presented in Figure 1(a). The three phases have an identical structure which consists of two arms and buffer inductors. The inductors named $L_{0}$ are used to suppress the circulating currents. The upper and the lower arm in each phase consist of $n$ series connected submodules, respectively. The configuration of submodules is shown in Figure 1(b). Each of the submodules is constructed with a half-bridge and a dc polar capacitor $C_{\mathrm{SM}}$. In this paper, the upper and the lower switching device of the half-bridge are named as S1 and S2, respectively.

By controlling the switching states of S1 and S2, the operating state of the submodule can be changed selectively. When S1 is switched on and S2 is switched off, the capacitor is put into the main circuit. The submodule is working as on-state, with its output voltage $u_{\mathrm{SM}}$ is equal to the capacitor voltage $u_{\mathrm{c}}$. When $\mathrm{S} 1$ is switched off and $\mathrm{S} 2$ is switched on, the capacitor is removed from the main circuit, and the submodule is working as off-state, with the $u_{\mathrm{SM}}$ is zero. The converter's phase voltages are defined as the voltages between the ac-side output points and the fictitious dc side neutral point " $o$ ". Phase voltage levels are related to the operating states of the submodules in each phase. Reference [24] analyzed the relationships between the maximum values of phase voltage levels and the numbers of submodules which are on. If the number of on-state submodules is maintained at $n$, the converter can generate $n+1$ voltage levels. $2 n+1$ voltage levels are also available when the number of on-state submodules varies among $n+1, n$ and $n-1$. The scope of this paper is restricted to the MMCs which generate $n+1$ phase voltage levels, but the strategy 
can be expended to the condition of $2 n+1$ voltage levels.
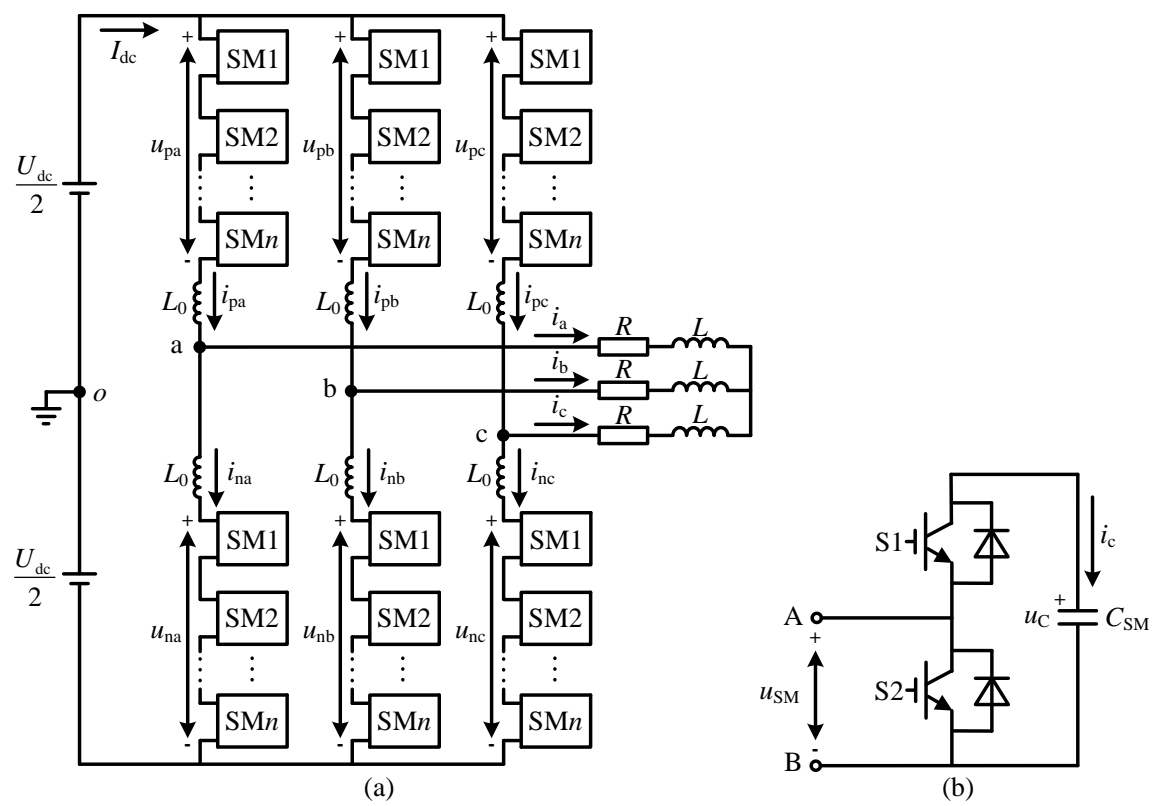

Figure 1. Circuit Configuration of the MMC and its SMS

\section{Mathematical Model of an MMC}

\subsection{Continuous Mathematical Model}

The positive directions of the electrical quantities are shown in Fig. 1. To facilitate the modeling, the model of the induction motor (IM) is replaced by a equivalent resistanceinductance load in this paper. Assuming that the capacitor voltages are ideally balanced, the upper and lower arm voltage $u_{\mathrm{p} j}$ and $u_{\mathrm{n} j}$ of phase $-j(j=\mathrm{a}, \mathrm{b}, \mathrm{c})$ can be expressed as

$$
\begin{aligned}
& u_{\mathrm{p} j}(t)=n_{\mathrm{p} j} \square \frac{U_{\mathrm{dc}}}{n} \\
& u_{\mathrm{n} j}(t)=n_{\mathrm{n} j} \square \frac{U_{\mathrm{dc}}}{n}
\end{aligned}
$$

where $n_{\mathrm{p} j}$ and $n_{\mathrm{n} j}$ represent the numbers of on-state submodules in the upper and lower arm of phase- $j$, respectively, and $U_{\mathrm{dc}}$ is the dc-link voltage. For the MMC of $n+1$ phase voltage levels, the sum of $n_{\mathrm{p} j}$ and $n_{\mathrm{n} j}$ is $n$.

In order to let each phase of MMC generate the expectative voltage levels at the ac-side, the specific numbers of submodules in the upper arms and the lower arms should be set as on-state. The relationship between the numbers and the output voltage is expressed by

$$
u_{j}(t)=\frac{u_{\mathrm{n} j}(t)-u_{\mathrm{p} j}(t)}{2}=\frac{n_{\mathrm{n} j}-n_{\mathrm{p} j}}{2 n} \square U_{\mathrm{dc}}
$$

where $u_{j}$ is the output phase voltage of phase $j$. It is obvious that $u_{j}$ changes stepwise from $-U_{\mathrm{dc}} / 2$ to $U_{\mathrm{dc}} / 2$ with the step of $U_{\mathrm{dc}} / n$.

Applying the Kirchhoff's current law (KCL) to phase-j, thus the arm currents can be described by

$$
\begin{aligned}
& i_{\mathrm{pj}}(t)=\frac{1}{2} i_{j}(t)+\frac{1}{3} I_{\mathrm{dc}}+i_{z j}(t) \\
& i_{\mathrm{nj}}(t)=-\frac{1}{2} i_{j}(t)+\frac{1}{3} I_{\mathrm{dc}}+i_{z j}(t)
\end{aligned}
$$

where $i_{\mathrm{p} j}$ and $i_{\mathrm{n} j}$ are the arm currents in phase-j, respectively, and $I_{\mathrm{dc}}$ is the dc-link 
current, and $i_{z j}$ are the circulating currents that flowing internally between the phases of the converter. Their positive directions are same with the arm currents and they never affect the output at the ac-side.

Based on equations (4) and (5), the ac-side phase current of phase- $j i_{j}$ is expressed as

$$
i_{j}(t)=i_{\mathrm{p} j}(t)-i_{\mathrm{nj}}(t)
$$

Similarly, applying the Kirchhoff's voltage law (KVL) to the phase- $j$ of MMC in Figure 1(a) yields

$$
\begin{aligned}
& \frac{U_{\mathrm{dc}}}{2}-u_{\mathrm{p} j}(t)-L_{0} \frac{\mathrm{d} i_{\mathrm{p} j}(t)}{\mathrm{d} t}=u_{j}(t) \\
& \frac{U_{\mathrm{dc}}}{2}-u_{\mathrm{n} j}(t)-L_{0} \frac{\mathrm{d} i_{\mathrm{nj}}(t)}{\mathrm{d} t}=-u_{j}(t) \\
& u_{j}(t)=R i_{j}(t)+L \frac{\mathrm{d} i_{j}(t)}{\mathrm{d} t}
\end{aligned}
$$

Subtracting equations (8) from (7), then substituting $i_{j}$ for $i_{\mathrm{p} j}, i_{\mathrm{n} j}$ and $\mathrm{u}_{j}$ based on equations (6) and (9). The dynamic character of ac-side phase current is described by

$$
u_{\mathrm{n} j}(t)-u_{\mathrm{p} j}(t)=L_{0} \frac{\mathrm{d} i_{j}(t)}{\mathrm{d} t}+2 R i_{j}(t)+2 L \frac{\mathrm{d} i_{j}(t)}{\mathrm{d} t}
$$

According to equations (10), the phase current has a tight relationship with $u_{\mathrm{p} j}$ and $u_{\mathrm{n} j}$. In other words, the phase current can be regulated by $u_{\mathrm{p} j}$ and $u_{\mathrm{n} j}$.

For the submodule in on-state of phase- $j$, the dynamic character of its capacitor voltage is expressed as

$$
i_{C r j i}(t)=C \frac{\mathrm{d} u_{C r j i}(t)}{\mathrm{d} t}
$$

where $r=\mathrm{p}, \mathrm{n}, i=1,2, \ldots, 2 n$, and $i_{\mathrm{Cp} j i}$ is equal to the upper arm current $i_{\mathrm{p} j}$ while $i_{\mathrm{Cn} j i}$ is equal to the lower arm current of phase- $j$.

\subsection{Discrete Mathematical Model for MPC Strategy}

The discrete mathematical models of the controlled variables need to be developed because the MPC strategy will use the models to predict the future values of the variables. So it is necessary to make the continuous mathematical models in Section 3.1 discretized.

Considering that the sampling period $T_{\mathrm{s}}$ is extremely short, the discretization process can be accomplished by the following Euler forward equation:

$$
\frac{\mathrm{d} x(t)}{\mathrm{d} t}=\frac{x(k+1)-x(k)}{T_{\mathrm{s}}}
$$

where $x(k+1)$ is the predictive value of $x$ at time $k+1$ and $x(k)$ is the actual value of $x$ at time $k$.

According to equations (10) and (12), the discrete mathematical model of phase current is deduced as

$$
i_{j}(k+1)=\frac{T_{\mathrm{s}}}{L_{0}+2 L}\left(u_{\mathrm{n} j}(k)-u_{\mathrm{p} j}(k)\right)+\left(1-\frac{2 T_{\mathrm{s}} R}{L_{0}+2 L}\right) i_{j}(k)
$$

where $i_{j}(k+1)$ is the predictive value of $i_{j}$ at time $k+1$ and $i_{j}(k)$ is the actual value of $i_{j}$ at time $k$.

Similarly, the discrete mathematical model of capacitor voltage in each submodule is deduced by equations (11) and (12):

$$
\left\{\begin{array}{l}
u_{C r j i}(k+1)=\frac{T_{\mathrm{s}}}{C_{\mathrm{SM}}} i_{C r j i}(k)+u_{C r j i}(k) \\
u_{C r j i}(k+1)=u_{C r j i}(k)
\end{array}\right.
$$


where $u_{\mathrm{C} r i j}(k+1)$ is the predictive value of $u_{\mathrm{C} r i i}$ at time $k+1$ and $u_{\mathrm{C} r i j}(k)$ is the actual value of $u_{\mathrm{C} r j i}$ at time $k$. If the submodule is in on-state at time $k$, the equations (14)-(a) is efficient. Else if the submodule is in off-state at time $k$, the equations (14)-(b) is efficient.

\section{Design of Control System}

\subsection{MPC Strategy for MMC}

Taking the submodules as switches that let the capacitors in or out of the circuit, there are $N=C_{2 n}^{n}$ switching states in each phase of MMC that generates $n+1$ voltage levels. Benefiting from the redundant switching states of each voltage level, the MPC strategy for MMC is feasible. The MPC controller will be designed for phase-j in this section.

4.1.1. Cost Function: The control objectives of the MPC controller are required as follows:

- make the ac-side phase currents of MMC track their reference values.

- keep the capacitor voltages balanced in each arm.

- maintain the arm energy balanced in each phase.

For the first control objective, the cost function $g_{1}$ should be defined as

$$
g_{1}=\left|i_{j}^{*}-i_{j}^{\mathrm{p}}\right|
$$

where $i_{j}^{*}$ is the reference current of phase- $j$ at ac-side and $i_{j}^{\mathrm{p}}$ is the predictive value of phase- $j$ current at ac-side.

To achieve the second control objective, the cost functions are deduced:

$$
\begin{aligned}
& g_{21}=\sum_{i=1}^{n}\left|u_{C r j i}{ }^{\mathrm{p}}-\frac{U_{\mathrm{dc}}}{n}\right| \\
& g_{22}=\sum_{i=2}^{n}\left|u_{C r j i} \mathrm{p}-u_{C r j 1} \mathrm{p}\right|
\end{aligned}
$$

where $u_{\mathrm{Cr} j i}{ }^{\mathrm{P}}$ is the predictive value of capacitor voltages in phase- $j$, and $u_{\mathrm{C} r j 1}{ }^{\mathrm{P}}$ is the predictive value of the first submodule's capacitor voltage in each arm of phase- $j$. The cost function $g_{21}$ makes the capacitor voltages approach their nominal value $U_{\mathrm{dc}} / \mathrm{n}$ as much as possible while $g_{22}$ keeps the voltages being the same from beginning to end.

The cost function $g_{3}$ for the third control objective is defined as

$$
g_{3}=\left|\sum_{i=1}^{n} u_{C \mathrm{p} j i}^{\mathrm{p}}-\sum_{i=1}^{n} u_{C \mathrm{nnji}}^{\mathrm{p}}\right|
$$

where $u_{\mathrm{Cp} j i}{ }^{\mathrm{P}}$ and $u_{\mathrm{Cnj} 1}^{\mathrm{P}}$ are the predictive value of capacitor voltages in the upper and lower arms, respectively. The cost function $g_{3}$ keeps the upper arm voltages and the lower arm voltage being the same to some degree. Thus, the energy of phase- $j$ is distributed in the upper and lower arms on average.

According to equations (15)-(18), the ultimate cost function in this paper is expressed as

$$
g=\left|i_{j}^{*}-i_{j}^{\mathrm{p}}\right|+\lambda_{1} \sum_{i=1}^{n}\left|u_{C r j i} \mathrm{p}-\frac{U_{\mathrm{dc}}}{n}\right|+\lambda_{2} \sum_{i=2}^{n}\left|u_{C r j i} \mathrm{p}^{\mathrm{p}}-u_{C r r 1} \mathrm{p}\right|+\lambda_{3}\left|\sum_{i=1}^{n} u_{C \mathrm{ppji}}^{\mathrm{p}}-\sum_{i=1}^{n} u_{C \mathrm{nnji}} \mathrm{p}\right|
$$

Where $\lambda_{1}, \lambda_{2}, \lambda_{3}$ are the weighting factors.

4.1.2 Minimization of Cost Function: The MPC block diagram of phase- $j$ of MMC is shown in Figure 2. The controlled variables will be predicted based on the predictive model in Section 3.2 for all available switching states, then the optimized switching state will be selected out by minimizing the cost function to drive the corresponding phase. The strategy can be described in the flow diagram in Figure 3. 


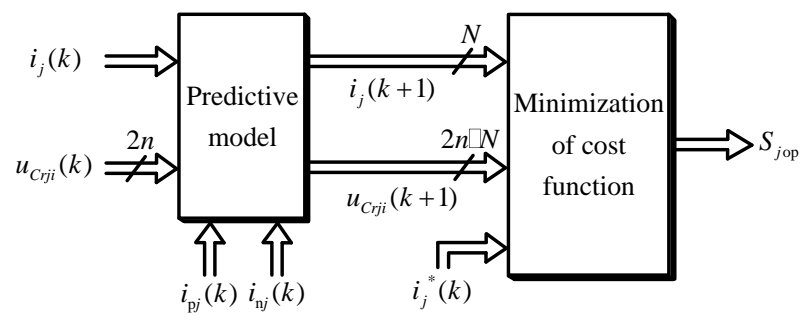

Figure 2. Model Predictive Control Block Diagram for Phase-J of MMC

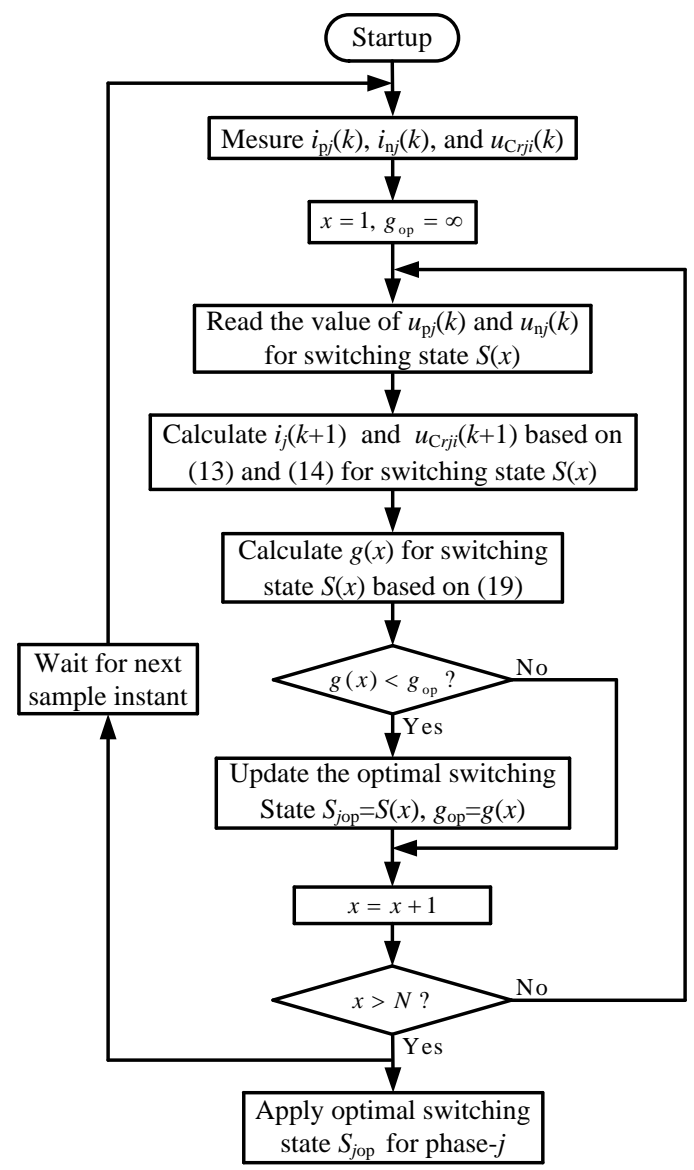

Figure 3. Flow Diagram of the MPC Strategy for Phase-j of MMC

\subsection{Model Predictive Vector Control}

Vector control is the most popular linear strategy in high performance electric motor drives. In this paper, a new rotor flux oriented vector control combined with MPC scheme is proposed for a MMC motor drive system which named model predictive vector control (MPVC).

The block diagram of the scheme for a MMC driving an induction motor is shown in Figure 4. In this scheme, the cascaded control loops are used to improve its performance. MPC controllers of each phase constitute the inner current control loop, which is formulated in the stationary abc reference frame. The rotor flux and rotational speed are regulated by two PI controllers which generate the reference currents $\left(i_{\mathrm{d}}{ }^{*}\right.$ and $\left.i_{\mathrm{q}}{ }^{*}\right)$ in the rotating dq reference frame. In this way, the current in d-axis $i_{\mathrm{d}}$ is related to the flux rotor and the current in q-axis $i_{\mathrm{q}}$ is related to the rotational speed. The angle of the three-phase stator currents is calculated by rotor flux observer for orientation of the rotating $\mathrm{dq}$ 
reference frame. By the dq- $\alpha \beta$ and the $\alpha \beta$-abc transforms, the reference currents $i_{\mathrm{d}}{ }^{*}$ and $i_{\mathrm{q}}{ }^{*}$ are transferred into $i_{\mathrm{a}}{ }^{*}, i_{\mathrm{b}}{ }^{*}$ and $i_{\mathrm{c}}{ }^{*}$ which are the reference currents of the MPC controllers. Then the MPC controller set the optimal switching states $S_{\text {aop }}, S_{\text {bop }}$ and $S_{\text {cop }}$ for the three phases of MMC based on the strategy designed in Section 4.1.

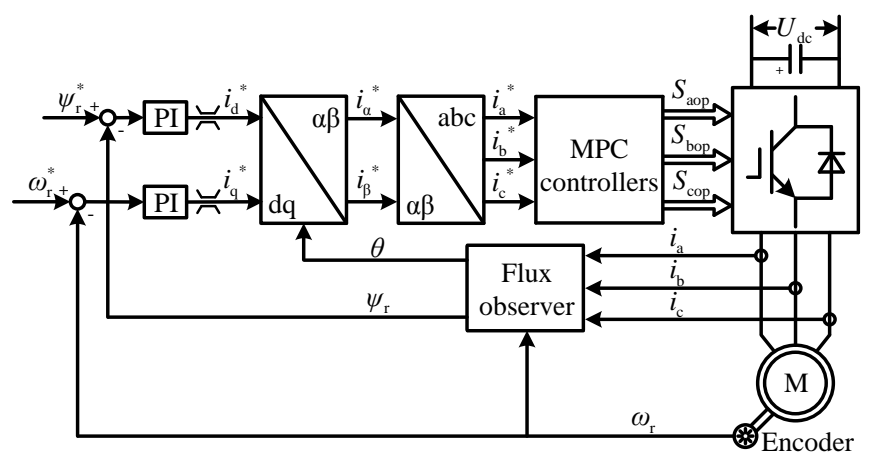

\section{Figure 4. Model Predictive Vector Control of A MMC Driving an IM}

Compared to the conventional vector control strategy, the conventional inner current control loops with liner PI regulators and pulse width modulation (PWM) module are replaced by MPC controllers in MPVC. Thus the difficulty of adjusting parameters in control system becomes easier since the PI regulators are abandoned.

\section{Performance Evaluation}

A five-level MMC based drive system with the proposed MPVC scheme described in section 4 is evaluated simulation analyses in the Matlab/Simulink software. Table 1 shows the parameters of the simulated system in Figures 1 and 4.

Table 1. Parameters of the Simulated System

\begin{tabular}{|c|c|c|}
\hline Parts & Parameters & Values \\
\hline MMC & $\begin{array}{l}\text { dc-link voltage } \\
\text { buffer inductance } \\
\text { No. of SMs per arm } \\
\text { SM capacitance } \\
\text { Nominal capacitor } \\
\text { voltage }\end{array}$ & $\begin{array}{l}U_{\mathrm{dc}}=650 \mathrm{~V} \\
L_{0}=6 \mathrm{mH} \\
n=4 \\
C_{\mathrm{SM}}=1880 \mu \mathrm{F} \\
u_{\mathrm{c}}^{*}=162.5 \mathrm{~V}\end{array}$ \\
\hline $\begin{array}{r}\mathrm{MPC} \\
\text { strategy }\end{array}$ & $\begin{array}{l}\text { equivalent resistance of } \\
\text { IM } \\
\text { equivalent inductance of } \\
\text { IM } \\
\text { sampling period }\end{array}$ & $\begin{array}{l}R=50 \Omega \\
L=50 \mathrm{mH} \\
T_{\mathrm{S}}=50 \mu \mathrm{s}\end{array}$ \\
\hline $\begin{array}{l}\text { inducti } \\
\text { on motor }\end{array}$ & $\begin{array}{l}\text { real power } \\
\text { rated voltage } \\
\text { rated frequency } \\
\text { rated rotating speed } \\
\text { stator resistance } \\
\text { stator inductance } \\
\text { rotor resistance } \\
\text { rotor inductance } \\
\text { mutual inductance } \\
\text { inertia }\end{array}$ & $\begin{array}{l}P_{\mathrm{m}}=2.2 \mathrm{~kW} \\
U_{\mathrm{m}}=380 \mathrm{~V} \\
f_{\mathrm{m}}=50 \mathrm{~Hz} \\
n_{\mathrm{m}}=1420 \mathrm{rpm} \\
R_{\mathrm{s}}=2.94 \Omega \\
L_{\mathrm{ls}}=0.02062 \mathrm{H} \\
R_{\mathrm{r}}=2.39 \Omega \\
L_{\mathrm{lr}}=0.02062 \mathrm{H} \\
L_{\mathrm{m}}=0.23507 \mathrm{H} \\
J=0.07 \mathrm{~kg} \cdot \mathrm{m}^{2}\end{array}$ \\
\hline
\end{tabular}


Simulation studies with no load and rated load are carried out to demonstrate the steady and dynamic characteristics of the drive system, respectively. In these studies, the start-up process of the IM is included and the capacitors of the submodules are initially with their nominal voltage value. Furthermore, the maximum torque of IM is limited at $30 \mathrm{~N} \cdot \mathrm{m}$, which is double of the rated value.

\subsection{Steady-State Performance}

\subsubsection{Unloaded Property}

The load of the IM is set to zero and the given rotating speed is equal to the rated value in the whole process of this condition. The system simulation waveforms are given in Figure 5. Figure 5(a) and (b) illustrate the actual rotating speed and the electromagnetic torque of the motor, respectively. The motor finishes starting up and steps into steady operation at about $t=0.4 \mathrm{~s}$. Afterwards, the rotating speed is $1420 \mathrm{rpm}$ and the electromagnetic torque is $0 \mathrm{~N} \cdot \mathrm{m}$.
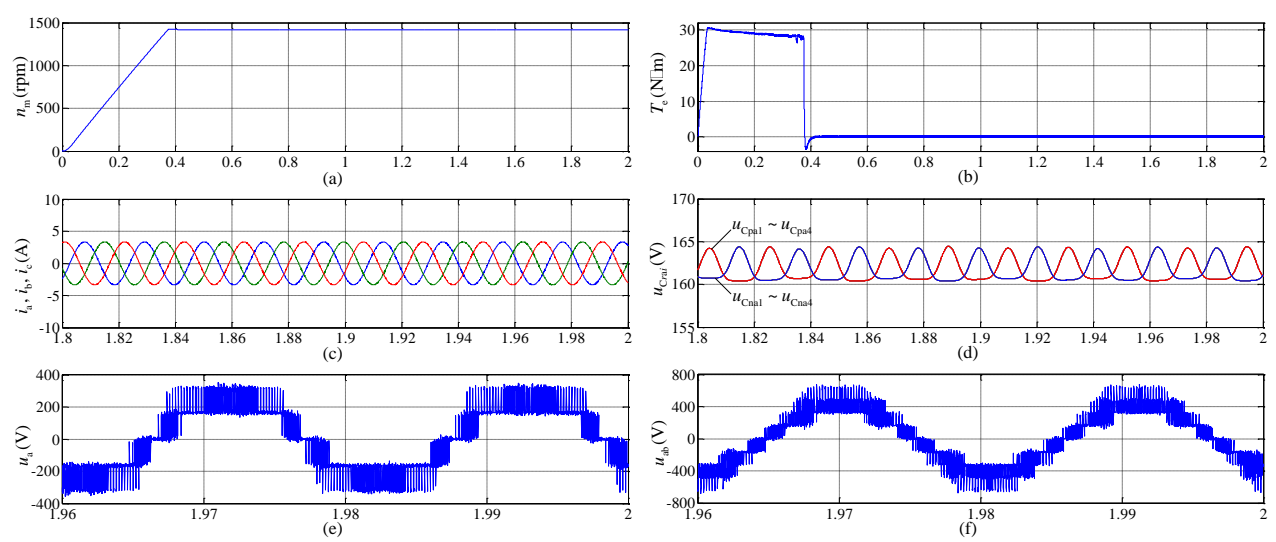

\section{Figure 5. Simulation Waveforms of the Steady Characteristic for the Drive System with No Load}

Figure 5(c) illustrates the stator currents of the motor and Figure 5(d) illustrates the capacitor voltages in phase-a during $1.8 \mathrm{~s}$ to $2 \mathrm{~s}$, respectively. As a result, the output currents of MMC are well sinusoidal and the capacitor voltages are maintained closed to the expected value $162.5 \mathrm{~V}$. The ripples of the capacitor voltages are limited to $4 \mathrm{~V}$. Figure 5(e) and (f) illustrate the phase-a output voltage and the phase-a\&b line-to-line voltage during $1.96 \mathrm{~s}$ to $2 \mathrm{~s}$, respectively. It is obvious that the voltage of phase-a has five voltage levels and the line-to-line voltage has nine voltage levels. The simulation results are consistent with theoretical analysis.

\subsubsection{Rated Load Property}

Both the load and the given rotating speed of the IM are set to their rated values in the whole process of this condition. The system simulation waveforms are given in Figure 6. Figure 6(a) and (b) illustrate the actual rotating speed and the electromagnetic torque of the motor, respectively. The motor finishes starting up and steps into steady operation at about $t=0.8 \mathrm{~s}$. Afterwards, the rotating speed is $1420 \mathrm{rpm}$ and the electromagnetic torque is $15 \mathrm{~N} \cdot \mathrm{m}$. 

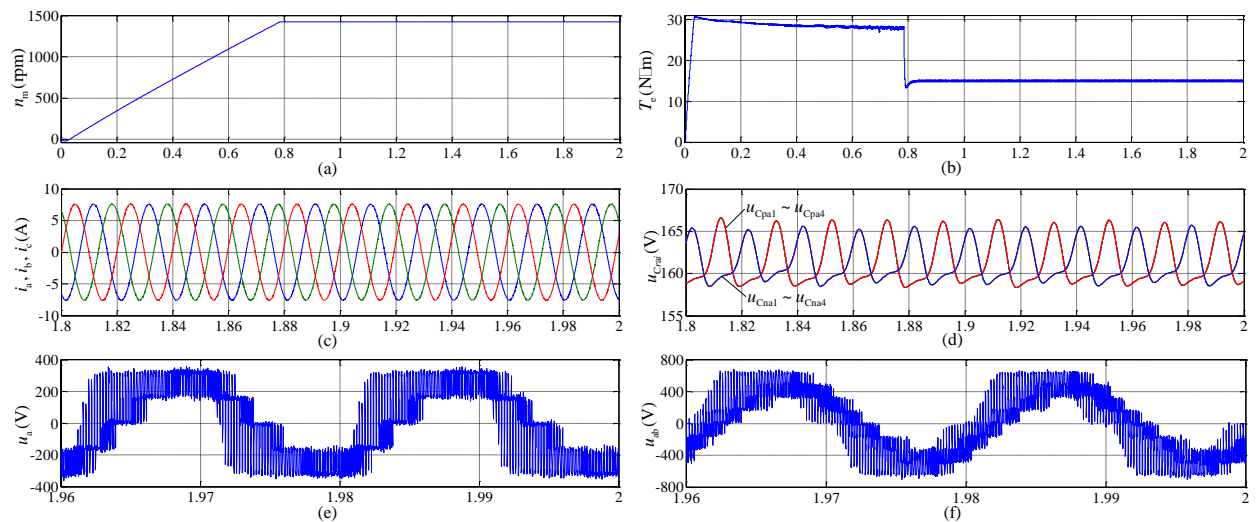

\section{Figure 6. Simulation Waveforms of the Steady Characteristic for the Drive System with Rated Load}

Figure 6(c) illustrates the stator currents of the motor and Figure 6(d) illustrates the capacitor voltages in phase-a during $1.8 \mathrm{~s}$ to $2 \mathrm{~s}$, respectively. Similar to the unloaded property, the currents are smoothed and the capacitor voltages are maintained closed to the expected value $162.5 \mathrm{~V}$. However, the amplitudes of the currents and the ripples of the capacitor voltages become larger with the increasing of the load. Though the ripples of the capacitor voltages increase to $8 \mathrm{~V}$, the effect of the capacitor balancing control is also satisfied. Figure 6(e) and (f) illustrate the phase-a output voltage and the phase-a\&b lineto-line voltage during $1.96 \mathrm{~s}$ to $2 \mathrm{~s}$, respectively. Both of them are irregular compared to the unloaded property, but it is also can be seen that the voltage of phase-a has five voltage levels and the line-to-line voltage has nine voltage levels.

From the simulations studied above, it can be known that for the submodules in the same arm, their voltages are kept as the same value all the time, and the energy of each arm in each phase is balanced in dynamic. The MPC controllers for the phases of MMC are correctly designed according the high-quality currents control and capacitor voltage balancing control.

\subsection{Dynamic-State Performance}

\subsubsection{Unloaded Property}

The load of the IM is set to zero in the whole process of this case. The given rotating speed is initially set to $710 \mathrm{rpm}$, which is half of the rated value. Then it is changed firstly to $1420 \mathrm{rpm}$ at $t=0.5 \mathrm{~s}$, then secondly to $-1420 \mathrm{rpm}$ at $t=1 \mathrm{~s}$ to cause a speed reversal of the motor. The corresponding system simulation waveforms are given in Figure 7.
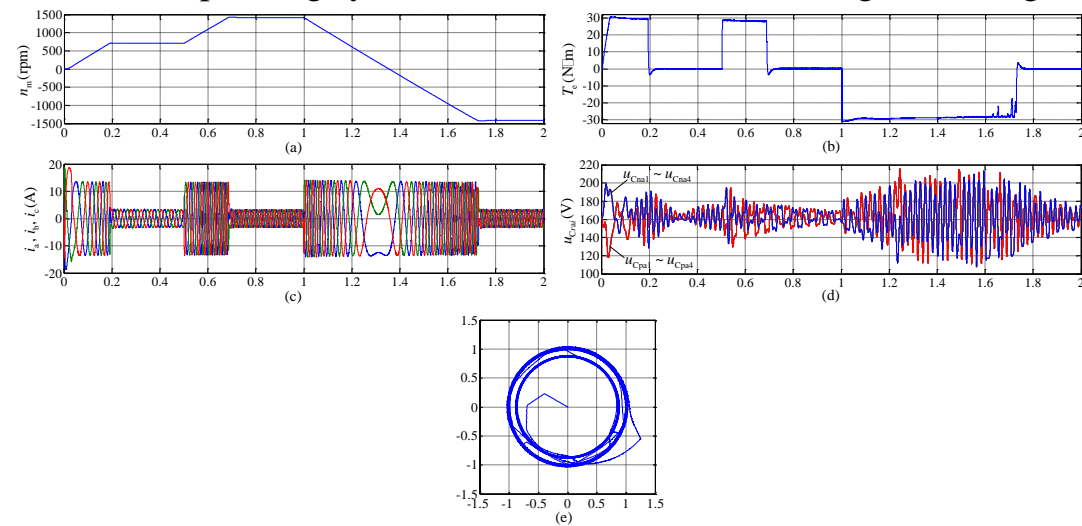

Figure 7. Simulation Waveforms of the Dynamic Characteristic for the Drive System with No Load 
Figure 7(a) and (b) illustrate the actual rotating speed and the electromagnetic torque of the motor, respectively. Figure 7(c) illustrates the stator currents and Fig. 7(d) illustrates the capacitor voltages in phase-a, respectively. Figure 7(e) illustrates the stator flux linkage locus in the rotating dq reference frame of the motor.

\subsubsection{Rated Load Property}

The load of the IM is set to rated value in the whole process of this case. The given rotating speed is initially set to $710 \mathrm{rpm}$. Then it is changed firstly to $1420 \mathrm{rpm}$ at $t=0.6 \mathrm{~s}$ and secondly to $-1420 \mathrm{rpm}$ at $t=1.2 \mathrm{~s}$ to cause a speed reversal of the motor. The corresponding simulation waveforms are illustrated in Figure 8.

Figure 8(a) and (b) illustrate the actual rotating speed and the electromagnetic torque of the motor, respectively. Figure 8(c) illustrates the stator currents and Figure 8(d) illustrates the capacitor voltages in phase-a, respectively. Figure 8(e) illustrates the stator flux linkage locus in the rotating dq reference frame of the motor.

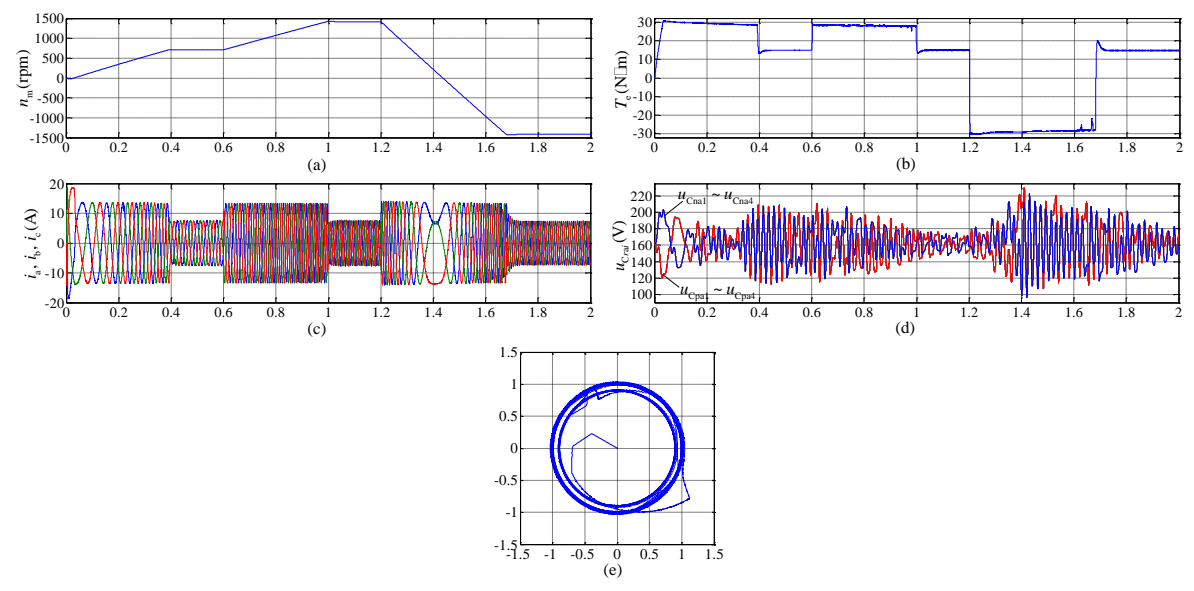

\section{Figure 8: Simulation Waveforms of the Dynamic Characteristic for the Drive System with Rated Load}

As can be seen, both the simulations with no load and rated load achieve nice dynamic performances. The actual rotating speed always tracks the given value accurately. Rapid responses to the step changes of given rotating speed are acquired by the currents and torque as their settling times are very short. Stator flux linkage loci are drawn in the the rotating $\mathrm{dq}$ reference frame demonstrte satisfactory characteristics of flux. However, consistent with the theoretical analysis, it's hard to make the capacitor voltages maintained their nominal value in the processes of speed adjustment. This is because, on one hand, the amplitudes of the output currents are always at the maximum value during the process, on the other hand, the output frequency varies across the low frequency segment. Thus, the ripples become large especially when the rotating speed is lower than $500 \mathrm{rpm}$. The capacitor voltages in the same arm are kept the as the same value, and the energy of each arm in each phase is balanced in dynamic, so the capacitor voltage balancing control is effective in these cases to some degree. What's more, the weighting factors of the MPC strategy can be enlarged to achieve a better control of capacitor voltages, but the control of output currents will be worse at the same time. It can be concluded that the MPC controllers make their efforts to ensure the high quality of output currents even though the capacitor voltages fluctuate in a very wide range. The proposed MPVC scheme is correct and effective for a high performance MMC drive system. 


\section{Conclusion}

This paper proposes an MPVC scheme for MMC electric motor drive systems. To apply MPC strategy into the controls of output currents and capacitor voltages for the inverter, discrete mathematical models of the controlled variables are presented based on the continuous mathematical models. The proposed MPVC scheme is constituted by using MPC controllers as parts of inner current control loops in rotor flux oriented vector control. Unlike the control approaches reported of MMC drive systems before in the literature, MPVC make it possible to control both the inverter and the machine in one computational stage with the cancellation of the independent modulation module.

The simulation analyses of a MMC drive with an induction motor are carried out by the Matlab/Simulik software. The simulation waveforms indicate that the drive system achieves high performances under various operating conditions regardless of the capacitor voltages' fluctuations at low speeds. Thus, it is possible to apply MMCs into high performance drive system by using MPVC, the following researches should be mainly concentrate on the capacitor voltage fluctuation suppressing control at low speeds.

\section{Acknowledgement}

The authors would like to thank the National Natural Science Foundation of China (51377160) and the Research and Innovation Program of Postgraduates in Jiangsu Province (KYLX_1384).

\section{References}

[1] J. Rodriguez, S. Bernet, B. Wu, J.O. Pontt and S. Kouro, "Multilevel voltage-source-converter topologies for industrial medium-voltage drives", IEEE Transactions on Industrial Electronics, vol. 54, no. 6, (2007), pp. 2930-2945.

[2] S. Kouro, M. Malinowski, K. Gopakumar, J. Pou, L. G. Franquelo, B. Wu, J. Rodriguez, M.A. Perez and J.I. Leon, "Recent advances and industrial applications of multilevel converters", IEEE Transactions on Industrial Electronics, vol. 57, no. 8, (2010), pp. 2553-2580.

[3] F.Z. Peng, W. Qian and D. Cao, "Recent advances in multilevel converter/inverter topologies and applications", in Proc. Conf. Rec. IEEE IPEC, (2010), June, pp. 492-501, Sapporo, Japan.

[4] A. Lesnicar and R. Marquardt, "An innovative modular multilevel converter suitable for a wide power range", presented at the IEEE Power Tech Conf., (2003), June, Bologna, Italy.

[5] S. Allebrod, R. Hamerski and R. Marquardt, "New transformer less, scalable modular multilevel converters for HVDC-transmission”, in Proc. IEEE Power Electron. Spec. Conf., (2008), June, pp. 174179, Rhodes, Australia.

[6] S. Rohner, S. Bernet, M. Hiller and R. Sommer, "Modulation, losses and semiconductor requirements of modular multilevel converters", IEEE Transactions on Industrial Electronics, vol. 57, no. 8, (2010), pp. 2633-2642.

[7] M. Hagiwara and H. Akagi, "Control and experiment of pulse width modulated modular multilevel converter", IEEE Transactions on Power Electronics, vol. 24, no. 7, (2009), pp. 1737-1746.

[8] S. Fazel, S. Bernet, D. Krug and K. Jalili, "Design and comparison of 4-kV neutral-point-clamped, flying-capacitor, and series-connected H-bridge multilevel converters", IEEE Transactions on Industry Applications, vol. 43, no. 4, (2007), pp. 1032-1040.

[9] M. Chaves, E. Margato, J. F. Silva, S. F. Pinto, and J. Santana, "Fast optimum-predictive control and capacitor voltage balancing strategy for bipolar back-to-back NPC converters in high-voltage direct current transmission systems", IET Generation, Transmission \& Distribution, vol. 5, no. 3, (2011), pp. 368-375.

[10] A. Ruderman, B. Reznikov and M. Margaliot, "Simple analysis of flying capacitor converter voltage balance dynamics for dc modulation," in Proc. EPE-PEMC, (2008), September, pp. 260-267, Poznan, Poland.

[11] S. Vazquez, J. Leon, J. Carrasco, L. Franquelo, E. Galvan, M. Reyes, J. Sanchez and E. Dominguez, "Analysis of the power balance in the cells of a multilevel cascaded H-bridge converter", IEEE Transactions on Industrial Electronics, vol. 57, no. 7, (2010), pp. 2287-2296.

[12] S. Rohner, S. Bernet, M. Hiller, and R. Sommer, "Modelling, simulation and analysis of a modular multilevel converter for medium voltage applications", in Proc. IEEE Int. Conf. Ind. Technol. (ICIT), (2010), March, pp. 775-782, Vi a del, Chile. 
[13] Z. Li, P. Wang, H. Zhu, Z. Chu and Y. Li, "An improved pulse width modulation method for choppercell-based modular multilevel converters", IEEE Transactions on Power Electronics, vol. 27, no. 8, (2012), pp. 3472-3481.

[14] K. Wang, Y. Li, Z. Zheng, and L. Xu, "Voltage balancing and fluctuation-suppression methods of floating capacitors in a new modular multilevel converter", IEEE Transactions on Power Electronics, vol. 60, no. 5, (2013), pp. 1943-1954.

[15] M. Hagiwara and H. Akagi, "Control and analysis of the modular multilevel cascade converter based on double-star chopper-cells (MMCC-DSCC)", IEEE Transactions on Power Electronics, vol. 26, no. 6, (2011), pp. 1649-1658.

[16] A. Korn, M. Winkelnkemper, and P. Steimer, "Low output frequency operation of the modular multilevel converter”, in Conf. Rec. IEEE ECCE, (2010), September, pp. 3993-3997, Atlanta, USA.

[17] J. Rodriguez, J. Pontt, C. A. Silva, P. Correa, P. Lezana, P. Cortes and U. Ammann, "Predictive current control of a voltage source inverter", IEEE Transactions on Industrial Electronics, vol. 54, no. 1, (2007), pp. 495-503.

[18] P. Cortes, P. Kazmierkowski, R. M. Kennel, D. E. Quevedo and J. Rodriguez, "Predictive Control in Power Electronics and Drives", IEEE Transactions on Industrial Electronics, vol. 55, no. 12, (2008), pp. 4312-4324.

[19] S. Kouro, P. Cortes, R. Vargas, U. Ammann and J. Rodriguez, "Model predictive control—a simple and powerful method to control power converters", IEEE Transactions on Industrial Electronics, vol. 56, no. 6, (2009), pp. 1826-1838.

[20] M.A. Perez, J. Rodriguez, E. Fuentes and F. Kammerer, "Predictive control of ac-ac modular multilevel converters”, IEEE Transactions on Industrial Electronics, vol. 59, no. 7, (2012), pp. 2832-2839.

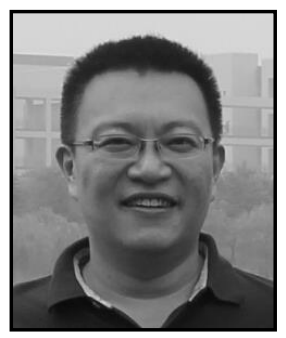

Peng Dai, He was born in HuaiBei, Anhui, China in 1973. He received his M.S. and Ph.D. in Electrical Engineering from the China University of Mining and Technology, China, in 1998 and 2006, respectively. He has been with the Department Electrical Engineering, China University of Mining and Technology, where he is currently a Professor. His current research interests include the stability and control of ac motor drives.

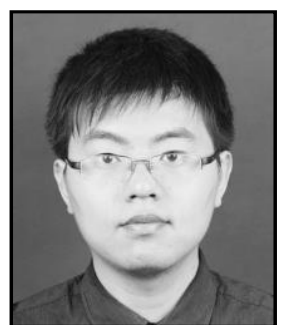

Zheng Gong, He was born in Tai'an, Shandong, China in 1990. He received his B.S. and M.S. from the China University of Mining and Technology, China, in 2012 and 2013, respectively. Since 2013, he has been working towards his Ph.D. in the Department of Information and Electrical Engineering, China University of Mining and Technology. His current research interests include the modeling and control of high power multilevel converters.

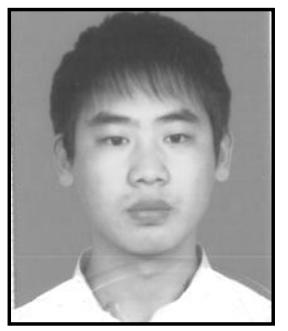

Guosheng Guo, He was born in Tai'an, Shandong, China in 1991. $\mathrm{He}$ received his B.S. from the China University of Mining and Technology, China in 2014. Since 2014, he has been working towards his M.S. in the Department of Information and Electrical Engineering, China University of Mining and Technology. His current research interests include the control of high power motor drives. 